\title{
Plasma MicroRNA-200c as A Prognostic Biomarker for Epithelial Ovarian Cancer
}

\author{
Addin Trirahmanto ${ }^{1}$, Hariyono Winarto ${ }^{1}$, Aria Kekalih², Ferry Sandra ${ }^{3, *}$ \\ ${ }^{1}$ Department of Obstetrics and Gynecology, Faculty of Medicine, Universitas Indonesia, Jl. Salemba Raya No.6, Jakarta, Indonesia \\ ${ }^{2}$ Department of Community Medicine, Faculty of Medicine, Universitas Indonesia, Jl. Salemba Raya No.6, Jakarta, Indonesia \\ ${ }^{3}$ Department of Biochemistry and Molecular Biology, Division of Oral Biology, Faculty of Dentistry, Universitas Trisakti, Jl. Kyai Tapa No.260, \\ Jakarta, Indonesia \\ *Corresponding author. E-mail: ferrysandra@gmail.com
}

Received date: Feb 20, 2019; Revised date: Jul 11, 2019; Accepted date: Jul 15, 2019

\section{Abstract}

$\mathrm{B}$ ACKGROUND: Ovarian cancer is the $8^{\text {th }}$ most prevalent cancer in women in the world. Current biomarker prognosis for ovarian cancer has numerous limitations, thus new biomarkers are needed. MicroRNAs (miRs) are considered as potential biomarkers in ovarian cancer as they are stable in blood. One candidate is miR-200c, the main regulator in epithelial transition to the mesenchyme. The aim of this study is to determine the role of miR-200c as prognostic biomarker for epithelial ovarian cancer (EOC).

METHODS: This is a prospective cohort study conducted at Dr. Sardjito Central General Hospital in Yogyakarta from September 2015 to July 2018. Sampling was done using consecutive sampling method. Forty plasma samples of EOC subjects were included in this study. miR-200c expression was quantified using Reverse Transcriptase
Quantitative Quantitative Polymerase Chain Reaction (RTqPCR) with miR-16 as the reference gene.

RESULTS: The expression of miR-200c was significantly higher in the group of subjects with preoperative CA-125 levels $>500 \mathrm{U} / \mathrm{mL}(p=0.009)$ than the group of subjects with preoperative CA-125 levels $<500 \mathrm{U} / \mathrm{mL}$. Subjects with higher miR-200c expression had lower survival rate than subjects with lower miR-200c expression, although not statistically significant.

CONCLUSION: The miR-200c could be a promising biomarker for EOC. Further studies with larger sample sizes are needed to clarify the prognostic value of miR200c.

KEYWORDS: miR-200c, epithelial ovarian cancer, prognosis, overall survival

Indones Biomed J. 2019; 11(3): 267-72

\section{Introduction}

Ovarian cancer is the third most prevalent cancer in women in Indonesia. Usually diagnosed in stage III-IV, ovarian cancer patient survival is generally low.(1) In a study performed at Dr. Cipto Mangunkusumo Central General Hospital, the 5 -year survival rate of epithelial ovarian cancer patients was $54.8 \%$.(2) There is barely any difference between incidence and mortality rate of ovarian cancer in developed and developing countries due to the fact that in all countries, $70 \%$ of patients were diagnosed in advanced stage.(3) Although new developments in detection and treatment of ovarian cancer are continually appearing, ovarian cancer is still the deadliest cancer in women. Determination of prognosis in ovarian cancer patients is an essential part of evaluation and therapy. Efforts to identify prognostic factors in epithelial ovarian cancer (EOC) continue to be sought in further research areas, including tumor characteristics, surgery, genetic patterns, immunological actors, and proteomic patterns.

Over the past ten years, scientific developments have changed the traditional concept of ovarian cancer. Ovarian cancer is currently not identified as a single disease, but rather 
diagnosed as a group of diseases with distinct morphologies and behaviors. About $80 \%$ of patients who have tumor residual disease after surgery and receive platinumbased combination chemotherapy, will have a median of 18-month disease-free survival rate.(4) Identification of factors related to disease aggressiveness can direct the development of new biomarkers and identification of targeted therapies that would greatly ameliorate the burden of this disease.(5)

MicroRNAs (miRs) are short RNA sequences which do not encode proteins but are the main expression regulators of various genes by acting on base pairs of 3'UTR mRNA targets that determine mRNA degradation or translation inhibition. The miRs are involved in the process of carcinogenesis, cell cycle, apoptosis, proliferation, invasion, metastasis, and chemoresistance. Their dysregulation is related with initiation and development of cancer in human, including ovarian cancer. miRs can act as onco-miR or as a tumor suppressor-miR. miRs are stable in blood circulation so they have potential as non-invasive biomarkers. Research on the role of miRs in tumorigenesis can provide a new paradigm for understanding ovarian cancer. The pattern of miR expression in primary tissue, circulation, and effusion fluid of ovarian cancer patients is not the only a potential marker in the detection and screening of non-invasive ovarian cancer, but can also be associated with survival, prognosis, and predictions of recurrence.(6)

miR-200c, a member of the miR-200 family, influences various types of cancer, including ovarian cancer. The miR-200c has been reported to play a role in epithelialmesenchymal transition (EMT), cancer stem cells (CSC), differentiation, modulation of cell division, apoptosis, and chemoresistance.(7) In this study, miR-200c in blood plasma is studied as a potential biomarker in determining prognosis in EOC.

\section{Methods}

\section{Subject Recruitment}

A prospective cohort study was conducted at Dr. Sardjito Central General Hospital in Yogyakarta from September 2015 to July 2018. Ethical clearance was granted from the Faculty of Medicine Universitas Gadjah Mada (KE/ FK/1085/EC/2015). Sixty-two subjects were recruited with consecutive sampling method. Subjects who suffer EOC, confirmed with clinical and histopathological examinations, were included, whereas subjects who had history of benign tumor and other cancer, were excluded. The EOC stage was classified based on The International Federation of Gynecology and Obstetrics/Fédération Internationale de Gynécologie et d'Obstétrique (FIGO).

\section{Sample Collection}

Pre-operative $5 \mathrm{~mL}$ peripheral venous blood was drawn in an ethylenediaminetetraacetic acid (EDTA) tube. Half of the blood was used to measure CA-125 level at Laboratory of Clinical Pathology, Dr. Sardjito Central General Hospital. And the other half was processed to get plasma for further analysis using Reverse Transcriptase Quantitative Polymerase Chain Reaction (RT-qPCR). After surgery, tumor was collected, prepared for hematoxylin-eosin staining and examined by pathologists.

\section{RT-qPCR}

RNA was isolated from plasma using miRCURY RNA Isolation Kit-Biofluids (Exiqon, Vedbaek, Denmark). Then total cDNA was prepared immediately from RNA using a Universal cDNA Synthesis kit (Exiqon). The qPCR was performed using miRCURY LNA Universal RT microRNA (Exiqon) with hsa-miR-16-5p LNA (Exiqon) and hsa-miR200c LNA primer sets (Exiqon). PCR was set for 40 cycles. miR-16 was used as the internal control. Analysis was carried out using GenEx 6 software to get results in the form of fold change and to measure the expressions of miR-200c. Data was processed using the CFX Manager 96 Biorad program to obtain the quantification cycle $(\mathrm{Cq})$.

\section{Survival Rate and Cox Regression}

Analysis of survival rate and Cox regression was done using SPSS version 23.0 (IBM Corp, New York, USA). Determination of high and low miR expressions was conducted using software R2: Genomic Analysis and Visualization Platform.

\section{Results}

From 62 subjects, 40 subjects were included in the study based on the inclusion and exclusion criteria. Most subjects were $>50$ years old $(52.5 \%)$ and had $\geq 1$ parity $(72.5 \%)$, early stage $(57.5 \%)$, histopathology type I $(60 \%),<1 \mathrm{~cm}$ residual tumor $(65 \%)$, and $<500 \mathrm{U} / \mathrm{mL}$ pre-operative CA$125(57.5 \%)$ (Table 1).

\section{The miR-200c Expression of EOC Subjects}

The expression of the miR-200c was shown in fold change compared to miR-16. The miR-200c fold changes (Figure 
Table 1. EOC subject characteristics.

\begin{tabular}{|c|c|c|}
\hline \multicolumn{2}{|c|}{ Category } & \multirow{2}{*}{$\begin{array}{c}\text { n (\%) } \\
19(47.5 \%)\end{array}$} \\
\hline Age (years) & $\leq 50$ & \\
\hline & $>50$ & $21(52.5 \%)$ \\
\hline \multirow[t]{2}{*}{ Parity } & Nulliparity & $11(27.5 \%)$ \\
\hline & Parity $\geq 1$ & $29(72.5 \%)$ \\
\hline \multicolumn{3}{|l|}{ EOC Stage } \\
\hline \multirow[t]{2}{*}{ Early Stage (Stage I-II) } & I & $19(47.5 \%)$ \\
\hline & II & $4(10 \%)$ \\
\hline \multirow[t]{2}{*}{ Late Stage III-IV } & III & $13(32.5 \%)$ \\
\hline & IV & $4(10 \%)$ \\
\hline \multicolumn{3}{|l|}{ Histopathology } \\
\hline \multirow[t]{4}{*}{ Type I } & Low-grade serous & $4(10 \%)$ \\
\hline & Clear cell & $5(12.5 \%)$ \\
\hline & Mucinous carcinoma & $14(35 \%)$ \\
\hline & Malignant Brenner tumor & $1(2.5 \%)$ \\
\hline \multirow[t]{4}{*}{ Type II } & High-grade serous & $12(30 \%)$ \\
\hline & High-grade endometrioid & $2(5 \%)$ \\
\hline & Undifferentiated carcinoma & $1(2.5 \%)$ \\
\hline & Carcinosarcoma & $1(2.5 \%)$ \\
\hline \multirow{2}{*}{ Residual Tumor After Surgery (cm) } & $<1$ & $26(65 \%)$ \\
\hline & $\geq 1$ & $14(35 \%)$ \\
\hline \multirow{2}{*}{ Pre-operative CA-125 Level (U/mL) } & $<500$ & $23(57.5 \%)$ \\
\hline & $\geq 500$ & $17(42.5 \%)$ \\
\hline
\end{tabular}

1) and $\triangle \mathrm{Cq}$ (Table 2) were analyzed in different age, parity, EOC stage, histopathology type, residual tumor, and CA-125 levels. A significant difference in mean of miR-200c $\Delta$ Cq between the group of pre-operative $<500$ $\mathrm{U} / \mathrm{mL}$ and $\geq 500 \mathrm{U} / \mathrm{mL}$ CA-125 was observed $(p=0.009)$ (Table 2).

\section{miR-200c and Overall Survival of EOC Subjects}

The overall survival was recorded for 3 months starting from the date of surgery. Among 40 subjects who were included in the study, 15 subjects died (35\%) and 25 people survived (65\%). Using R2 Genomic Analysis and Visualization Platform, low and high miR-200c groups were classified and then analyzed using the Kaplan-Meier method for overall survival. Using log-rank test, low expression of miR-200c was shown to be associated with higher overall survival, although not statistically significant (Table 3, Figure 2).

\section{Discussion}

Most of the EOC subjects in this study were in the stage I-II $(57.5 \%)$, which is different from the majority of studies whose subjects were $60-70 \%$ in the stage III-IV.(1) This might be related with the fact that the majority of the subjects of this study were having histopathology type I (60\%). The results of this study are not much different from studies which found that the most common histopathology type was serous carcinoma, followed by mucinous and endometrioid carcinoma. $(8,9)$ The EOC type I grows slowly, usually in stage I-II, rarely has a p53 mutation and tends to be resistant to platinum-based chemotherapy.(10)

The miR-200c expression of the group with preoperative CA-125 level $>500 \mathrm{U} / \mathrm{mL}$ was significantly higher $(p=0.009$ ) than the group with $<500 \mathrm{U} / \mathrm{mL}$. Although not significant, miR-200c expression was higher in the 
$\mathbf{A}$

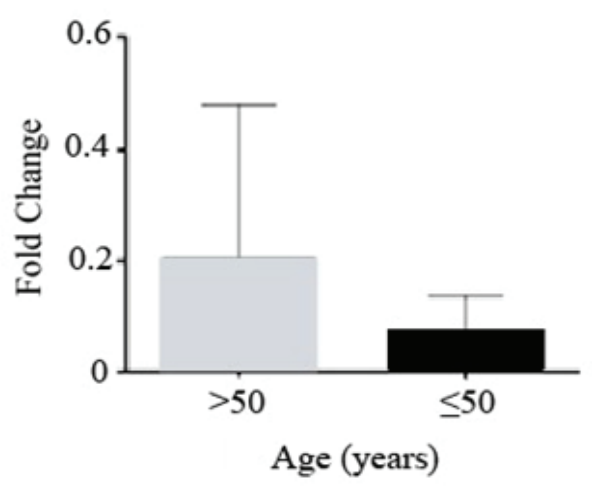

C

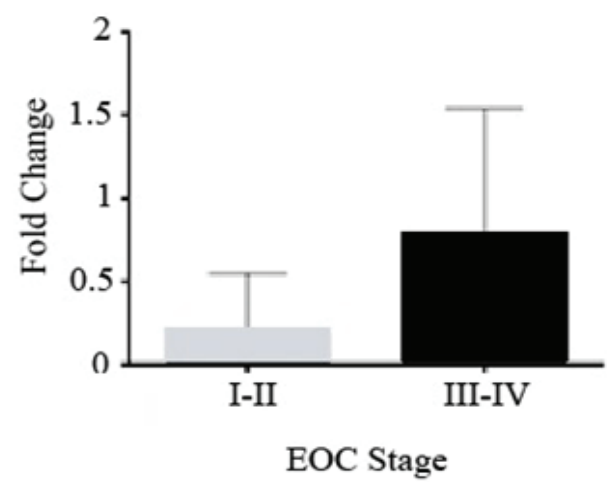

$\mathbf{E}$

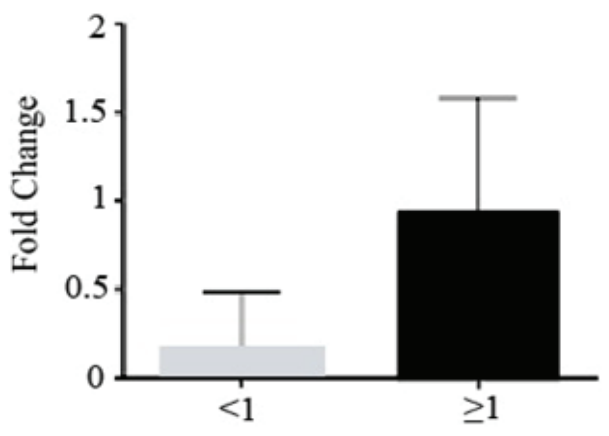

Residual Tumor $(\mathrm{cm})$
B

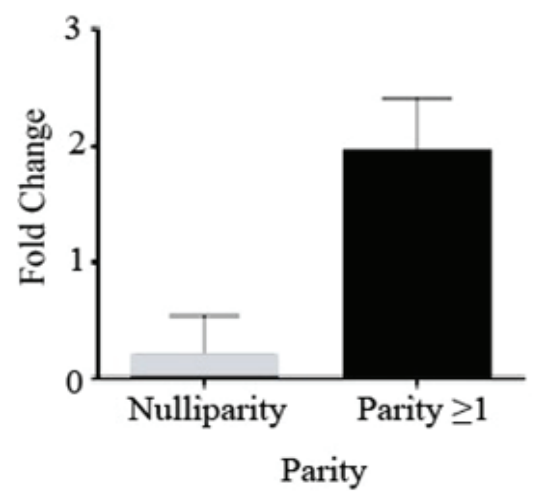

D

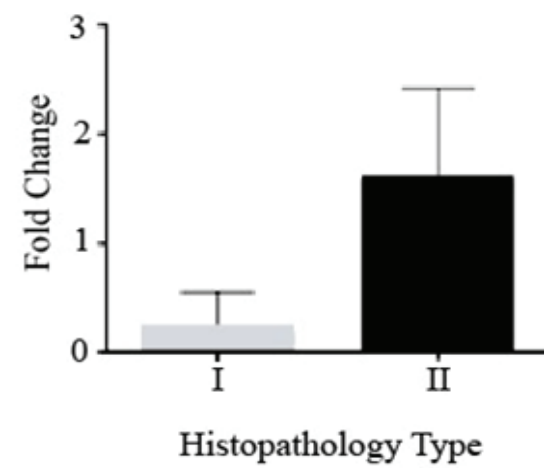

$\mathbf{F}$

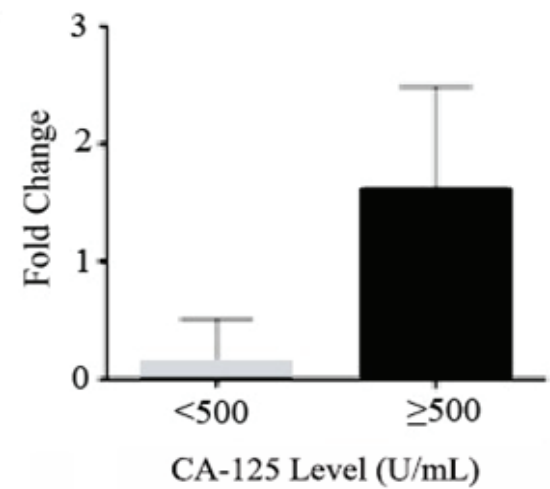

Figure 1. miR-200c fold change of EOC subjects. The expression of miR-200c gene was shown in fold change compared to miR-16. miR-200c fold changes of EOC subjects were grouped in different age (A), parity (B), EOC stage (C), histopathology type (D), residual tumor (E) and preoperative CA-125 level (F).

EOC histopathology type II than in the type I. miR-200c expressions was also higher in EOC Stage I-II than Stage III-IV. The increase of miR-200c expression in this study showed a tendency towards severity of EOC. This is in agreement with the previous study, which found a significant difference in the miR-200c expression between EOC stage III-IV and healthy controls.(11)
Some studies stated that higher expression level of the miR-200 family was associated with decrease in patient's survival, while in other studies the opposite effect of high miR-200 expression has been reported. The exact reason underlying these contradictory findings is still not clear. One report shows that miR-200c can act as a suppressor or trigger for aggressive phenotypes, depending on the location 
Table 2. The miR-200c $\Delta \mathrm{Cq}$ of EOC subjects.

\begin{tabular}{llc}
\hline \multicolumn{1}{c}{ Category } & $\begin{array}{c}\text { Mean of } \\
\text { miR-200c } \Delta \mathbf{C q}\end{array}$ & $\boldsymbol{p}$-value \\
\hline Age (years) & & \\
$\leq 50$ & $0.01 \pm 1.18$ & 0.76 \\
$>50$ & $0.20 \pm 2.63$ & \\
\hline Parity & & \\
Nulliparity & $0.01 \pm 2.07$ & \\
Parity $\geq 1$ & $1.19 \pm 2.00$ & \\
\hline EOC Stage & & 0.37 \\
Stage I-II & $0.01 \pm 1.37$ & \\
Stage III-IV & $1.36 \pm 2.74$ & \\
\hline Histopathology & & \\
Type I & 0.16 \\
Type II & $0.01 \pm 1.32$ & \\
\hline Residual Tumor After Surgery (cm) & & \\
$<1$ & $0.01 \pm 2.11$ & \\
$\geq 1$ & $0.97 \pm 1.99$ & \\
\hline Pre-ope rative CA-125 Level (U/mL) & & \\
$<500$ & & \\
\hline 500 & & \\
\hline & & \\
\hline
\end{tabular}

of the Human antigen R (HuR).(12) In current study, after bivariate analysis using log-rank test and Kaplan-Meier curve, it was found that high miR-200c expression had lower overall EOC patient survival, but not statistically significant. This result suggests a possible oncomiR role of miR-200c in EOC. The finding of miR-200c as an oncomiR has been reported in serous ovarian cancer, the high expression of miR-200c had a worse median overall survival compared to the one of low miR-200c expression.(13)
The relationship between the high expression of the miR-200 family and the prognosis of cancer is still controversial. It has been shown in a meta-analysis report that high expression of the miR-200 family in various tumors was associated with a low overall survival. The expression of the miR-200 family is associated with a poor prognosis of various types of cancer, implying the potential of the family miR-200 family for cancer prognosis in clinical practice.(14)

Table 3. miR-200c expressions and overall survival of EOC subjects.

\begin{tabular}{lccccc}
\hline $\begin{array}{c}\text { Level of miR-200c } \\
\text { Expression }\end{array}$ & $\mathbf{n}$ & Mortality & $\begin{array}{c}\text { Hazard Ratio } \\
(\text { HR) }\end{array}$ & $\begin{array}{c}\text { Confidence } \\
\text { Interval (CI) 95\% }\end{array}$ & $\boldsymbol{p}$-value \\
\hline Low & 11 & 2 & 1 & $0.638-12.543$ & 0.167 \\
High & 29 & 13 & 1.908 & & \\
\hline
\end{tabular}




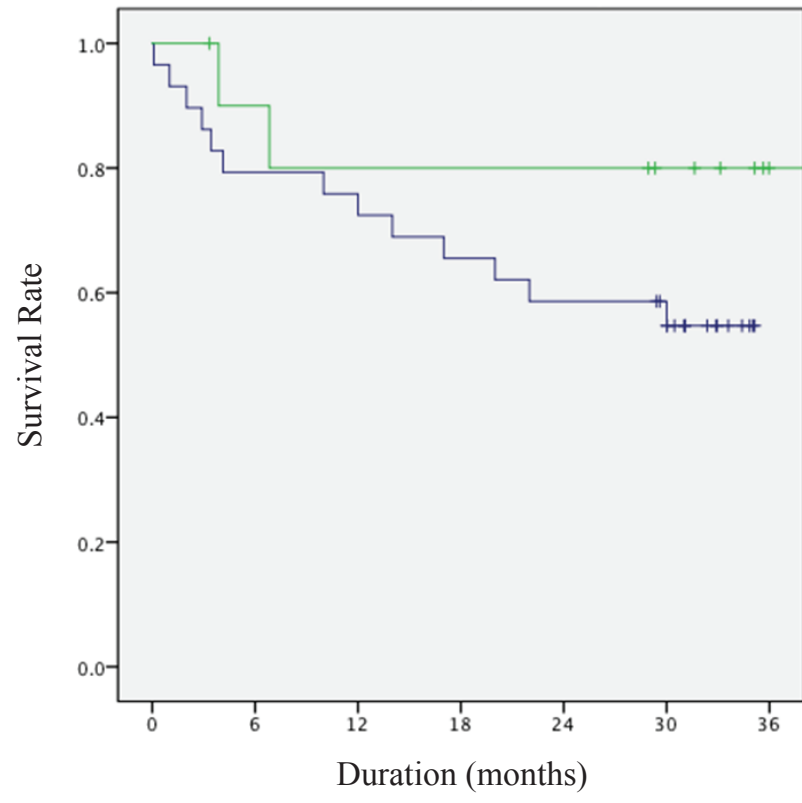

\section{Conclusion}

The miR-200c expression is correlated with the severity in EOC. Higher expression of miR-200c correlate with worse overall survival, although not statistically significant. miR200c could be a promising biomarker for EOC. Further studies with larger sample sizes are needed to clarify the prognostic value of miR-200c.

\section{References}

1. Bray F, Ferlay J, Soerjomataram I, Siegel RL, Torre LA, Jemal A. Global cancer statistics 2018: GLOBOCAN estimates of incidence and mortality worldwide for 36 cancers in 185 countries. CA Cancer J Clin. 2018; 68: 394-424.

2. Noela F, Nuryanto KH. Epidemiology data of ovarian cancer in Dr. Cipto Mangunkusumo Hospital, Jakarta. Indones J Obstet Gynecol. 2016; 4: 101-6.

3. Mutch DG, Prat J. 2014 FIGO staging for ovarian, fallopian tube and peritoneal cancer. Gynecol Oncol. 2014; 133: 401-4.

4. Herzog TJ, Spetzler D, Xiao N, Burnett K, Maney T, Voss A, et al. Impact of molecular profiling on overall survival of patients with advanced ovarian cancer. Oncotarget. 2016; 7: 19840-9.

\section{Level of miR-200c}

Expression

\section{High \\ Low

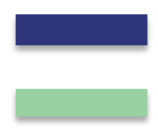

Figure 2. Survival rate of subjects with different miR-200c expression levels.

5. Taylor PT, Haverstick D. Re: New guidelines to evaluate the response to treatment in solid tumors (ovarian cancer). J Natl Cancer Inst. 2005; 97: 151.

6. Kinose Y, Sawada K, Nakamura K, Kimura T. The Role of microRNAs in ovarian cancer. Biomed Res Int. 2014; 2014: 249393. doi: $10.1155 / 2014 / 249393$.

7. Feng X, Wang Z, Fillmore R, Xi Y. MiR-200, a new star miRNA in human cancer. Cancer Lett. 2014; 344: 166-73.

8. Jiang X, Tang H, Chen T. Epidemiology of gynecologic cancers in China. J Gynecol Oncol. 2018; 29: e7. doi: 10.3802/jgo.2018.29.e7.

9. Wentzensen N, Poole EM, Trabert B, White E, Arslan AA, Patel AV, et al. Ovarian cancer risk factors by histologic subtype: an analysis from the ovarian cancer cohort consortium. J Clin Oncol. 2016; 34 : 2888-98.

10. Kurman RJ, Shih IEM. The dualistic model of ovarian carcinogenesis: revisited, revised, and expanded. Am J Pathol. 2016; 186: 733-47.

11. Chi DS, Eisenhauer EL, Zivanovic O, Sonoda Y, Abu-Rustum NR, Levine DA, et al. Improved progression-free and overall survival in advanced ovarian cancer as a result of a change in surgical paradigm. Gynecol Oncol. 2009; 114: 26-31.

12. Prislei S, Martinelli E, Mariani M, Raspaglio G, Sieber S, Ferrandina $\mathrm{G}$, et al. MiR-200c and HuR in ovarian cancer. BMC Cancer. 2013; 13: 72. doi: 10.1186/1471-2407-13-72.

13. Nam EJ, Yoon H, Kim SW, Kim H, Kim YT, Kim JH, et al. MicroRNA expression profiles in serous ovarian carcinoma. Clin Cancer Res. 2008; $14: 2690-5$.

14. Liu W, Zhang K, Wei P, Hu Y, Peng Y, Fang X, et al. Correlation between miR-200 Family Overexpression and Cancer Prognosis. Dis Markers. 2018; 2018: 6071826. doi: 10.1155/2018/6071826. 